\title{
Shining a spotlight on somatic mutations in ulcerative colitis
}

\section{Cf \\ both studies \\ identify \\ mutations in \\ genes related \\ to IL-17 \\ signalling in \\ the context \\ of ulcerative \\ colitis}

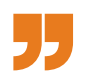

Two new studies published in Nature shed light on somatic mutations that occur in ulcerative colitis, providing insights into mutagenesis in the inflamed intestinal epithelium and mutational profiles in colitis-associated cancer compared with non-dysplastic tissue. Interestingly, both studies identify mutations in genes related to IL-17 signalling in the context of ulcerative colitis.

In the first study, the researchers used intensive multi-regional sampling to examine clonal expansion in the intestinal epithelia of patients with ulcerative colitis and those without the disease as controls. "Clonal expansion in apparently normal or non-cancer tissues is one of the hottest topics in the field of cancer research," says author Seishi Ogawa. "We wanted to understand how chronic inflammation shapes tissue remodelling and how it correlates with cancer development, in terms of driver mutations associated with such remodelling."

To understand the dynamics of ulcerative colitis, Ogawa and colleagues used whole-exome sequencing (WES) to evaluate somatic mutations from single intestinal crypts, before performing WES of bulk crypt samples. They found that the inflamed intestine in ulcerative

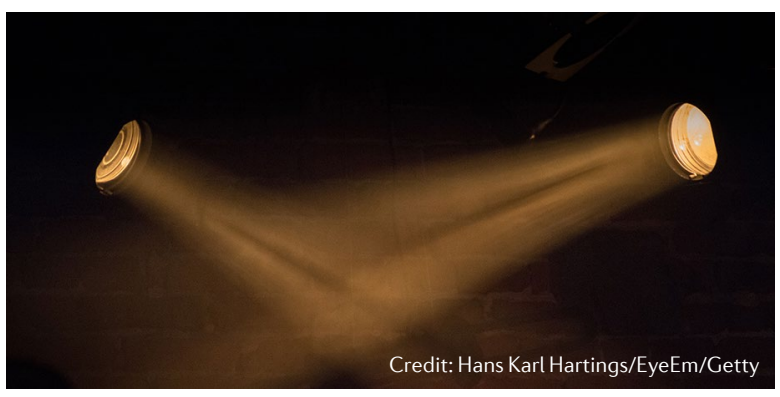

colitis undergoes widespread remodelling by pervasive clones. Moreover, the majority of these clones were positively selected by acquiring mutations that frequently involved genes implicated in the downregulation of IL-17 and other pro-inflammatory signals (such as NFKBIZ, PIGR and ZC3H12A). Furthermore, mutational profiles varied substantially between colitis-associated cancer and non-dysplastic tissues in ulcerative colitis.

Examining the NFKBIZ mutants in more detail, Ogawa and colleagues found that mutations in NFKBIZ were highly prevalent in the epithelium of patients with ulcerative colitis, but rarely found in sporadic or colitis-associated colorectal cancer. Importantly, tumour formation was markedly reduced in Nfkbiz-mutant mice and disruption of NFKBIZ in human colorectal cancer cells compromised cell competition. "Mutations in NFKBIZ and $\mathrm{ZC} 3 \mathrm{H} 12 \mathrm{~A}$ are highly prevalent in the epithelium of patients with ulcerative colitis but rarely found in colitis-associated cancer, indicating that NFKBIZ-mutant or $\mathrm{ZC} 3 \mathrm{H} 12 \mathrm{~A}$-mutant cells are selected against during colorectal carcinogenesis, which could potentially be exploited for therapeutics in colorectal cancer," adds Ogawa.

In the second study, the researchers used WES of 76 human colon organoids to identify a unique pattern of mutagenesis in the inflamed intestinal epithelium during ulcerative colitis. "It was unclear how fast normal colonic stem cells acquire somatic mutations throughout life because of the technical difficulty in accurately measuring somatic variants in single stem cell clones," explains author Toshiro Sato. This limitation has now been overcome with the development of organoid technology, and Sato and colleagues used this approach to study mutation rates in ulcerative colitis.

The researchers found that the ulcerative colitis epithelium accumulated somatic mutations in multiple genes related to IL-17 signalling (including $N F K B I Z$, $P I G R$ and $Z C 3 H 12 A$, which are rarely affected in colon cancer), and targeted sequencing validated the pervasive spread of these mutations.

Importantly, CRISPR-based knockout screening in colon organoids revealed that the mutations conferred resistance to the proapoptotic response induced by IL-17A, implying that IL-17A is detrimental for the intestinal epithelium and that clones with IL-17A pathway mutations can avoid IL-17A-mediated cytotoxicity. Further experiments using NFKBIZknockout organoids indicated that inducible nitric oxide synthase mediates this IL-17A-induced apoptosis and drives the subsequent selective expansion of IL-17Aresistant mutant clones in ulcerative colitis tissue. "The unique somatic mutation pattern in ulcerative colitis epithelium showed the first evidence of environment-driven genetic evolution in human tissues," reports Sato.

Both groups of investigators are planning further research to understand how somatic mutations in the ulcerative colitis epithelium can influence disease activity in a bid to improve our knowledge of the pathogenesis of inflammatory bowel disease.

Katrina Ray

ORIGINAL ARTICLE Kakiuchi, N. et al. Frequent mutations that converge on the NFKBIZ pathway in ulcerative colitis. Nature 577, 260-265 (2020) | Nanki, K. et al. Somatic inflammatory gene mutations in human ulcerative colitis epithelium. Nature 577, 254-259 (2020) 